\title{
CHARACTERIZATION OF BEAM DYNAMICS IN THE APS INJECTOR RINGS USING TIME-RESOLVED IMAGING TECHNIQUES
}

\author{
B. X. Yang, A. H. Lumpkin, M. Borland, K. Harkay, A. Nassiri \\ Argonne National Lab, 9700 South Cass Avenue, Argonne, IL 60439, USA
}

Abstract

Images taken with streak cameras and gated intensified cameras with both time (longitudinal) and spatial (transverse) resolution reveal a wealth of information about circular accele ators. We illustrate a novel technique by a sequence of dual-sweep streak camera images taken at a high dispersion location in the booster synchrotron, where the horizontal coordinate is strongly correlated with the particle energy and the "top-view" of the beam gives a good approximation to the particle density distribution in the longitudinal phase space. A sequence of top-view images taken right after injection clearly shows the beam dynamics in the phase space. We report another example from the positron accumulator ring for the characterization of its beam compression bunching with the 12th harmonic rf.

\section{INTRODUCTION}

The time-resolved imaging techniques using gated cameras and dual-sweep streak cameras have been applied to linacbas $-\mathrm{d}$ free-electron lasers and large accelerators $[1,2]$. In this work, we will show that by viewing the optical synchrotron radiation from a high dispersion area with a streak camera, one can directly visualize particle motion in the longitudinal phase space. In another case, we report studies of longitudinal damping and bucket impurity in the APS positron accumulator ring (PAR).

\section{VISUALIZING THE LONGITUDINAL PHASE SPACE IN THE APS BOOSTER SYNCHROTRON}

\subsection{Conditions in the APS Booster Synchrotron}

Three synchrotron radiation ports have been installed in the Advanced Photon Source (APS) booster synchrotron; this experiment was performed at the third port, which has the highest dispersion. Table 1 lists values of relevant parameters for the accelerator and fully damped beam at this location. While the size of the beam is dominated by the emittance effect for fully damped beams, any momentum offset (from the nominal value) due to injection or ramping phase/energy mismatch will cause beam motions in the longitudinal phase space. This is particularly true in the first half of the ramp (acceleration) where the damping by synchrotron radiation is weak.

Tabie 1 APS Booster Synchrotron Parameter at Photon Port No. 3 (horizontal beta function $=10.65 \mathrm{~m}$ )

\begin{tabular}{lcc}
\hline Particle energy $(\mathrm{GeV})$ & 0.375 & 7.0 \\
& (inject) & (extract) \\
Design emittance $(\mathrm{mm} \cdot \mathrm{mrad})$ & 0.25 & 0.13 \\
Horizontal size due to emittance $(\mathrm{mm})$ & 1.632 & 1.181 \\
Horizontal size due to energy spread(mm) & 0.683 & 0.683 \\
\hline
\end{tabular}

\subsection{Experimental Setup [3]}

The visible synchrotron radiation light was collected by a molybdenum mirror, relayed by an achromatic lens, and focused onto the streak camera slits by a second lens. The part of the slits being illuminated is thus a true image of $t] ;:$ horizontal profile of the particle beam.

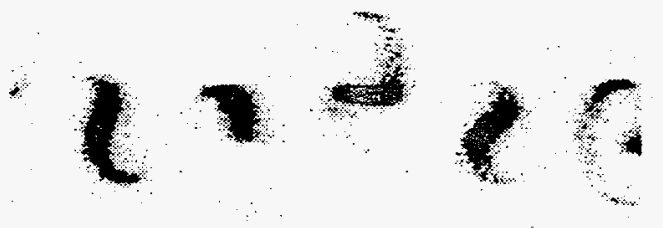

(a)

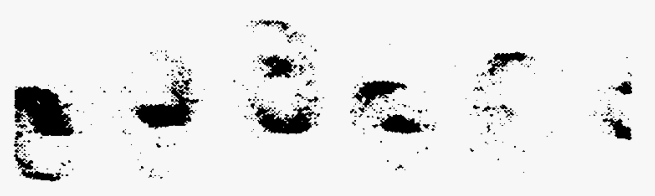

(c)

Figure 1: Top view of the positron bunch in the APS booster synchrotron at a high dispersion area. All pictures are taken with vertical full scale of $5 \mathrm{~ns}$ and horizontal $50 \mu \mathrm{s}$. (a) the first $50 \mu \mathrm{s}$ after injection, (b) after $50 \mu$ s delay from the injection; (c) after $100 \mu$ s delay.

A dual-sweep streak camera (Hamamatsu C5680 with M5677 slow-sweep unit and M5679 dual-time base extender unit) was used to record the images. A DG535 delay generator (from Stanford Research), triggered by the booster injection

The submitted manuscript has been ereated by the University of Chicago as Operator of Argonne National Laboratory ("Argonne") under Contract No. W-31-109-ENG-38 with the U.S. Department of Energy. The L.S. Government retains for itself, and others acting on its behalf, a paid-up, nonexclusive, irrevocable worldwide license in said article to reproduce, prepare derivatrve works. distribute copies to the public, and pertorm publicly and display publicly, by or on behulf of the Government. 


\section{DISCLAMMER}

Portions of this document may be illegible in electronic image products. Images are produced from the best available original document. 


\section{DISCLAIMER}

This report was prepared as an account of work sponsored by an agency of the United States Government. Neither the United States Government nor any agency thereof, nor any of their employees, makes any warranty, express or implied, or assumes any legal liability or responsibility for the accuracy, completeness, or usefulness of any information, apparatus, product, or process disclosed, or represents that its use would not infringe privately owned rights. Reference herein to any specific commercial product, process, or service by trade name, trademark, manufacturer, or otherwise does not necessarily constitute or imply its endorsement, recommendation, or favoring by the United States Government or any agency thereof. The views and opinions of authors expressed herein do not necessarily state or reflect those of the United States Government or any agency thereof. 
timing pulse, was used to generate the trigger for the horizontal scan unit M5679.

The positron revolution time in the booster is $1.228 \mu$ s. The turn signal was used to trigger a second delay generator which generated a vertical trigger pulse for the vertical scan unit M5677 in multiples of the revolution time. The frequency of the vertical trigger pulse was chosen to be about $6-10$ times that of the horizontal scan time of the camera since only 6 - 10 beam images could fit in the same screen without interfering with each other.

\subsection{Results and Discussion}

Figure 1 shows three frames taken after the injection, $50 \mu \mathrm{s}$ (40 turns) apart in their delay time. The interval between each beam image is seven turns. The vertical axis corresponds to time or particle phase, and the horizontal motion is the combination of the particle's betatron motion and energy offset. The pictures strow that the synchrotron motion is the dominant component. After injection, the captured bunch tumbles (clockwise in the picture) in the phase space with a period $\sim 25 \mu \mathrm{s}$, which roughly correlates to the measured synchrotron f- quency of $30 \mathrm{kHz}$ about $1 \mathrm{~ms}$ into the ramp. The motion the particle immediately starts to decohere due to nonlinearity of the effective potential, decohering almost completely into a "doughnut" shape within the first $150 \mu \mathrm{s}$ (125 turns).
Figure 2 shows two frames taken in the same experiment but using a larger time scale: the horizontal extent is now $50 \mathrm{~ms}$, and the frames are $50 \mathrm{~ms}$ apart in delay time. The size of the "doughnuts" shrinks down with the ramping, since increased particle energy results in increased synchrotron radiation and enhanced longitudinal damping. The emittance effect finally dominates the beam size at $\sim 100 \mathrm{~ms}$ into the ramp or when the positron energy reaches $\sim 3 \mathrm{GeV}$.

\section{GATED CAMERA MEASUREMENT OF APS BOOSTER SYNCHROTRON BEAM EMITTANCE}

Due to continued energy change of the particle beam during the ramp in the booster, the beam profile imaging is best performed with only a single pass to avoid blurring due to beam motion. We used an intensified gated camera (Quik 05 from Stanford Computer Optics) to acquire the image. The camera was triggered by a delayed pulse from the injection timing, and its exposure time was set to be less than the revolution time. Figure 3 shows a typical image taken about five turns before the extraction. Using the design lattice functions, we deduced from the beam profile $\left(\sigma_{x}=0.80 \mathrm{~mm}\right.$, $\sigma_{y}=0.17 \mathrm{~mm}$ ) that the emittance at extraction was $\varepsilon=0.125 \mathrm{~mm} \cdot \mathrm{mrad}$, with a vertical coupling of $\chi=0.02$, matching the design goal of $\varepsilon=0.131 \mathrm{~mm} \cdot \mathrm{mrad}$, and $\chi<0.1$.

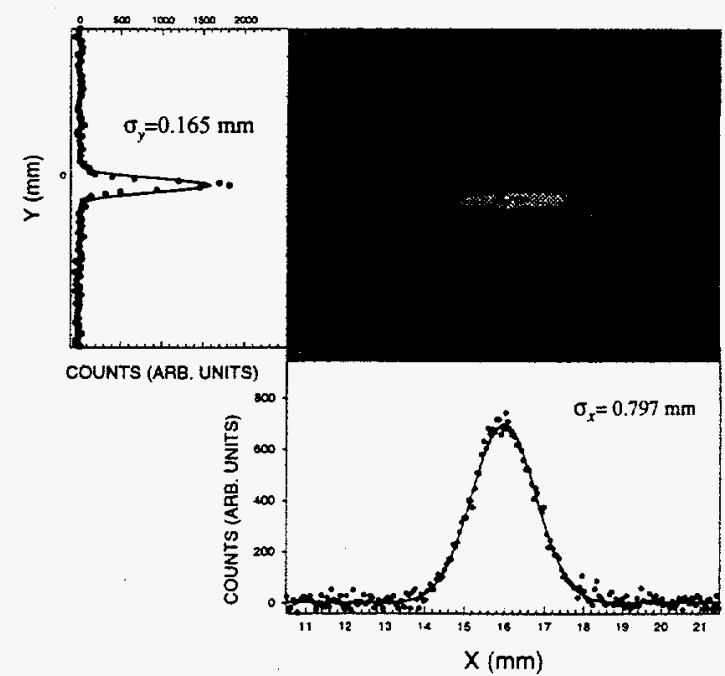

Figure 3: The front view of the positron bunch in the APS booster synchrotron at the extraction. a snapshot taken in a single pass.

\section{STUDIES OF BEAM DAMPING IN THE PAR}

In the APS positron accumulator ring (PAR), the bunch length is compressed before the extraction by turning on a twelfth harmonic if cavity (RF-12, $117.3 \mathrm{MHz}$ ) for about $100 \mathrm{~ms}$ to match the booster capture requirement. The phase of the harmonic relative to the fundamental is crucial to the compression.

We used a streak camera to study the beam dynamics during the compression. The optical transport [4] here is similar to that in the booster. except that a short focal length lens was used to condense the photons so that the spatial information was lost. Figure 4 show's a streak image taken
Figure 2: Top view of the positron bunch in the APS booster synchrotron at a high dispersion area. Both pictures were taken with vertical full scale of $5 \mathrm{~ns}$ and horizontal $50 \mathrm{~ms}$. (a) the first $50 \mathrm{~ms}$ after injection and (b) after $50 \mathrm{~ms}$ delay from the injection. 
with $50 \mathrm{~ns}$ full scale in the vertical direction, and $50 \mathrm{~ms}$ in the horizontal. The vertical scan is triggered every $2 \mathrm{~ms}$. The RF12 cavity was turned on about $7 \mathrm{~ms}$ after the start of the horizontal scan in this picture. By carefully examining this picture one can extract much information about the compression process.

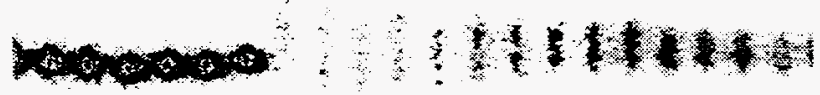

Figure 4: A dual-sweep image of the APS PAR beam during bunch compression by the $12^{\text {th }}$ harmonics if $(117.3 \mathrm{MHz})$.

\subsection{Bunch Length and Longitudinal Damping}

The bunch length can be obtained by fitting the integrated vertical intensity profile of any of the beam images. The bunch with $R F-12$ off was found to be 0.84 ns long, which compares with a theoretical value of $0.88 \mathrm{~ns}$ for the operating condition during the experiment. Figure 5 plots the bunch length as a function of time after RF-12 was turned on. From this plot, we deduced the longitudinal damping time to be $24.1 \mathrm{~ms}$, which may be compared with its design value of $25.4 \mathrm{~ms}$. The bunch length at the end of the compression was measured in a similar manner and found to be $0.22 \mathrm{~ns}$, which may be compared with its design value of $0.21 \mathrm{~ns}$.

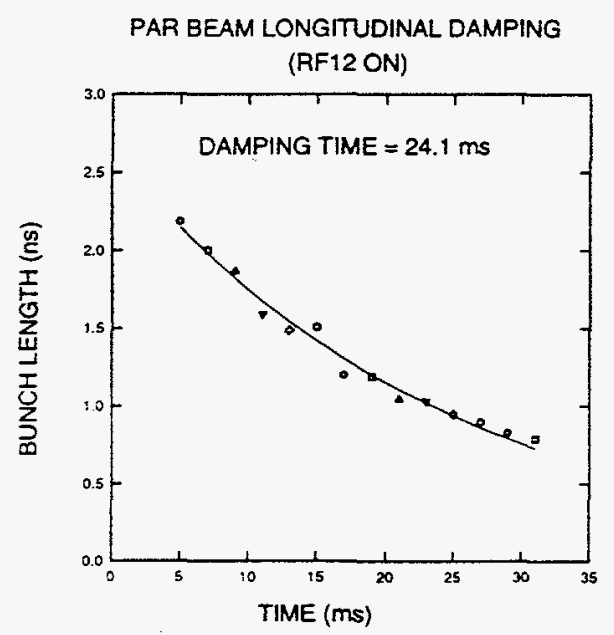

Figure 5: Bunch length of the PAR as a function of time after the $12^{\mathrm{t}}$ harmonic is turned on.

\subsection{Bunch Purity and Compressor Phase}

It can also be seen from Fig. 4 that the phase of the harmonic cavity was mismatched when this image was taken. The mismatch caused the bunch to lengthen significantly after turning on RF-12. In fact, some particles were spilled over to the adjacent bucket and captured. This satellite bunch propagated through the booster synchrotron into the storage ring (known as bucket impurity), where it became quite a nuisance for nuclear resonance experiments.

Figure 6 shows the fraction of the integrated intensity of the main and satellite peaks as a function of RF-12 phase. By varying the phase, one can minimize the satellite bunch intensity to a level acceptable to the users.

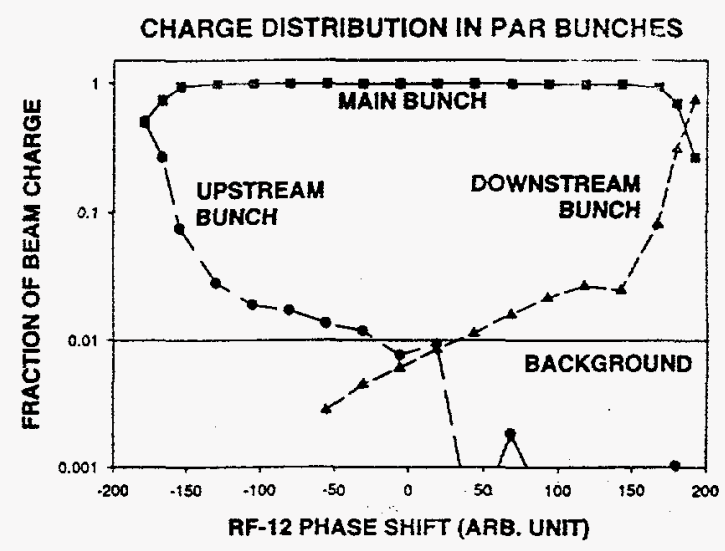

Figure 6: Intensity of bunches as a function of the RF-12 phase. The optimal operating phase shift is near zero.

\section{SUMMARY}

The time-resolved imaging techniques have been used successfully to study the beam dynamics in the APS injector accelerator. A novel technique was used to visualize the evolution of particle bunches in the longitudinal phase space. The technique is useful in optimizing injection phase/energy match for circular machines. It will also be useful in studying bunch compression dynamics for the fourth-generation light sources.

\section{ACKNOWLEDGEMENTS}

This work was supported by U. S. Department of Energy, Office of Basic Energy Sciences, under Contract No. W-31109-ENG-38.

\section{REFERENCES}

[1] A. H. Lumpkin, "Advanced, time-resolved imaging techniques for electron-beam characterizations," AIP Conf. Proc. 229, 1991.

[2] E. Rossa, "Real time single shot three-dimensional measurement of the picosecond photon bunches," AIP Conf. Proc. 333, 1995.

[3] A. H. Lumpkin and B. Yang, "Status of the radiation monitors for the APS facility rings." Proc. of the 1995 PAC, 1995.

[4] W. Berg, B. Yang, A. Lumpkin and J. Jones, "Design and commissioning of the photon monitors and optical transport lines for the Advanced Photon Source positron accumulator ring," AIP Conf. Proc. 333, 1995. 